\title{
Measurements of the gingival papillae architecture using cone-beam computed tomography in young Chinese adults
}

\author{
Gang Yang ${ }^{1}$, Jie Cao ${ }^{1}$, Wenjie Hu ${ }^{\text {Corresp., }}{ }^{,}$, Kwok-Hung Chung ${ }^{2}$ \\ ${ }^{1}$ Department of Periodontology, Peking University School and Hospital of Stomatology, National Clinical Research Center for Oral Disease, National \\ Engineering Laboratory for Digital and Material Technology of Stomatology, Beijing Key Laboratory of Digital Stomatology, Beijing, China \\ Department of Restorative Dentistry, University of Washington, Seattle, Washington, United States \\ Corresponding Author: Wenjie Hu \\ Email address: huwenjie@pkuss.bjmu.edu.cn
}

Background. The aim of this study was to measure the morphology of interdental papillae (IP) in maxillary anterior teeth using cone-beam computed tomography (CBCT).

Methods. Twenty-seven periodontally healthy subjects with 135 IP were evaluated by means of periodontal examination and a CBCT scan with an elastomeric matrix containing radiopaque material in position. According to the status of tooth contact and presence of IP, subjects were categorized into three groups: open contact point, complete papillae, and deficient papillae group. The papillae height (PH), facial-lingual thickness (FLT), and interdental distance (IDD) were measured. Data was analyzed with the significance level at $\alpha=0.05$.

Results. The mean PH values were $4.17 \pm 0.51 \mathrm{~mm}, 3.99 \pm 0.61 \mathrm{~mm}$, and $3.99 \pm 0.62 \mathrm{~mm}$, for the open contact group, complete papilla, and deficient papilla group, respectively. The PH values of the recorded sites among central incisors, lateral incisors, and canine were $4.13 \pm 0.56 \mathrm{~mm}, 3.87 \pm 0.63 \mathrm{~mm}$, and $4.07 \pm 0.58 \mathrm{~mm}$, respectively. No statistically significant differences of the PH values were determined between the above three tested groups as well as between the different sites $(p>0.05)$. The IDD values obtained from the complete papilla group were significantly lower than the other tested groups ( $p<$ 0.05).

Conclusion. The contact point condition of upper anterior sextants may not influence the presence and dimension of the IP in periodontally healthy subjects. 
1 Measurements of the gingival papillae architecture using cone-beam 2 computed tomography in young Chinese adults

3

Gang Yang ${ }^{1}$, Jie Cao ${ }^{1}$, Wenjie $\mathrm{Hu}^{1}$, Kwok-Hung Chung ${ }^{2}$

${ }^{1}$ Department of Periodontology, Peking University School and Hospital of Stomatology, National Clinical Research Center for Oral Disease, National Engineering Laboratory for Digital and Material Technology of Stomatology, Beijing Key Laboratory of Digital Stomatology, Beijing, China

2 Department of Restorative Dentistry, University of Washington, Seattle, Washington, United States

Corresponding Author:

Wenjie $\mathrm{Hu}^{1}$

22 Zhongguancun Avenue South, Haidian District, Beijing 100081, PR China.

Email address: huwenjie@pkuss.bjmu.edu.cn

\section{Abstract}

Background. The aim of this study was to measure the morphology of interdental papillae

(IP) in maxillary anterior teeth using cone-beam computed tomography (CBCT).

Methods. Twenty-seven periodontally healthy subjects with 135 IP were evaluated by means of periodontal examination and a $\mathrm{CBCT}$ scan with an elastomeric matrix containing radiopaque material in position. According to the status of tooth contact and presence of IP, subjects were categorized into three groups: open contact point, complete papillae, and deficient papillae group. The papillae height (PH), facial-lingual thickness (FLT), and interdental distance (IDD) were measured. Data was analyzed with the significance level at $\alpha=0.05$.

Results. The mean PH values were $4.17 \pm 0.51 \mathrm{~mm}, 3.99 \pm 0.61 \mathrm{~mm}$, and $3.99 \pm 0.62 \mathrm{~mm}$, for the open contact group, complete papilla, and deficient papilla group, respectively. The $\mathrm{PH}$ values of the recorded sites among central incisors, lateral incisors, and canine were $4.13 \pm 0.56 \mathrm{~mm}$, 
$313.87 \pm 0.63 \mathrm{~mm}$, and $4.07 \pm 0.58 \mathrm{~mm}$, respectively. No statistically significant differences of the

$32 \mathrm{PH}$ values were determined between the above three tested groups as well as between the different

33 sites $(\mathrm{p}>0.05)$. The IDD values obtained from the complete papilla group were significantly

34 lower than the other tested groups $(\mathrm{p}<0.05)$.

Conclusion. The contact point condition of upper anterior sextants may not influence the presence and dimension of the IP in periodontally healthy subjects.

\section{Introduction}

Interdental papilla (IP), which occupies the interdental embrasure, not only acts as a barrier in protecting the periodontal structures, but also plays a critical role in facial esthetics (Chow et al., 2010; Chen et al., 2010; Kim et al., 2011). Preserving the gingival tissue in the embrasures of the maxillary anterior sextant is a key consideration in periodontal, restorative, and implant treatment (Tarnow, Magner, \& Fletcher, 1992; Kolte, Kolte, \& Mishra, 2014). Various factors may influence the presence or absence of the IP including the distance between underlying bone crest (BC) and contact point (CP), interdental distance (IDD), periodontal phenotype, and adjacent tooth form (Tarnow, Magner, \& Fletcher, 1992; Cho et al., 2006; Chen et al., 2010; Chow et al., 2010; Kim et al., 2011). Among these factors, the CP-BC distance has been considered as a dominant factor. According to the previous report, the papilla was often completely present when the CP-BC distance was $\leq 5 \mathrm{~mm}$ (Tarnow, Magner, \& Fletcher, 1992). Moreover, it has been reported that the positioning of proximal contact coronally during prosthetic treatment may cause loss of the IP (Nozawa et al., 2011). Previous studies indicate that the IDD is a crucial determinant of PH (Cho et al., 2006; Kim et al., 2011). The FLT of the base of each papilla was suggested as a potential factor influencing the presence of the IP (Gastaldo, Cury, \& Sendyk, 2004; Chang, 2008). Previously, there were several studies reported the relationship between the presence and the 
54 dimensional size of the gingival architecture using conventional techniques (Chow et al., 2010; de

55 Santana, de Miranda, \& de Santana, 2017).

56 Cone-beam computed tomography (CBCT) has been widely used in clinical examination and

57 evaluation of the hard tissue in periodontal and implant treatment (Scarfe, 2006; Vera, et al., 2012).

58 It has been reported that one of the shortcomings of CBCT is its inability to discriminate soft

59 tissues that has rendered CBCT an exclusive tool for hard tissue imaging (Guerrero et al., 2006).

60 The soft tissues of the lips and cheeks collapse on the facial gingiva and the tongue occupies the 61 space of the space of the oral cavity, thus completely preventing the visualization of the soft tissues 62 of the periodontium (Januario, Barriviera, \& Duarte, 2008). In fact, CBCT can be used to measure

63 the dimensions of gingival tissue when soft tissue retraction or impression material with opaque 64 agent is applied to separate the gingiva and other soft tissues (Januario, Barriviera, \& Duarte, 2008; Cao et al., 2015). Thus, the CBCT imaging can visualize and measure both hard and soft tissues of periodontium and dentogingival attachment apparatus accurately. The clinicians will 67 also be capable of determining the relationships among structures of periodontium such as the gingival margin, bone crest, and the cementoenamel junction, as well as measuring the width of 69 facial and palatal/lingual alveolar bone and gingiva. This will aid clinicians in the planning and execution of periodontal and implant procedures with increased predictability. The dimension of 71 facial and palatal/lingual alveolar bone and gingiva had been measured by previous study (Januario, Barriviera, \& Duarte, 2008; Cao et al., 2015; Ogawa et al., 2020), while the architecture of the IP has not been visualized and measured by CBCT imaging yet. The aim of this

74 investigation was to assess the spatial architecture of the IP in the periodontally healthy young Chinese adults using CBCT. The null hypothesis was that no significant correlation would be 76 found among different tooth contact types and presence of IP. 


\section{Materials \& Methods}

78 Sample selection

79 This study was approved by the Biomedical Ethics Committee of Peking University School and 80 Hospital of Stomatology (approval ID PKUSSIRB-2012047) and was conducted in accordance

81 with the Helsinki Declaration of 1975, as revised in 2013. Written informed consent was obtained

82 from the participants in accordance with the guidelines of the committee for the subject selection

83 process. The recruitment of research subjects was conducted and enrolled among 486 dental

84 students and staff under preventive care screened at the School of Stomatology. The selection

85 criteria included, (i) Chinese adults of 20 years or older with fully erupted permanent dentitions,

86 (ii) no medications known to increase the risk of gingival hyperplasia, (iii) no pregnancy, (iv)

87 grossly healthy maxillary anterior gingival tissue with probing depths $\leq 3 \mathrm{~mm}$ and gingival index

88 of grades 0 to $1,(\mathrm{v})$ well-aligned maxillary anterior teeth, (vi) absence of gingival recession, (vii)

89 no previous surgical periodontal treatments in the anterior maxillary region, (viii) no artificial

90 crowns and anterior restorations, (ix) no missing teeth in the maxillary anterior region. Twenty-

91 seven periodontally healthy subjects including 12 males and 15 females were recruited with the

92 mean age of 24.5 years old. All subjects were treated with supra-gingival scaling in the Department

93 of Periodontics 2 weeks before clinical examination and the CBCT imaging.

94 Clinical examination

95 All proximal contacts and spaces between the right and left maxillary canine were examined 96 clinically using dental floss (Oral-B) and explorer (Hu-Friedy) clinically. Each papilla was given

97 a papilla score (PS) of 0-3 based upon Nordland and Tarnow's classification system (Nordland $98 \&$ Tarnow, 1998). The papilla was defined as complete (PS $=0)$ or deficient (PS $\geqslant 1)$. The open 
99 contact case was defined with no resistance by passing a dental floss through the height of contours

100 of two adjacent teeth. There are three types of the relationship between CP and IP categorized and

101 grouped as the open contact area group, the deficient papilla group, and the complete papilla group

102 (Figure 1).

103 CBCT examination

104 The CBCT scanning procedure was performed as previously described (Cao et al., 2015). Briefly,

105 a matrix, including mesial sides of the maxillary first premolars, was fabricated in the mouth

106 individually using silicone impression material (RAPID putty, Coltène/Whaledent AG). The

107 thickness of the silicone matrix was approximately $6 \mathrm{~mm}$, with at least $8 \mathrm{~mm}$ of silicone material

108 extending apically from the free gingival margins facially and palatally. After a 4-minute initial

109 set intraorally, the silicone matrix was removed from the patient's mouth, and the intaglio surface

110 was trimmed evenly with a scraper to remove a layer approximate $1 \mathrm{~mm}$ of silicone material from

111 the surface opposing the mucogingival region of the matrix in order to create approximately $1 \mathrm{~mm}$

112 of relief space. The putty-wash impression technique was applied, and the final impression was

113 made using a mixture of barium sulfate powder (Reagent grade, Qingdao Dongfeng Chemical)

114 and alginate impression material (Heraeus Kulzer) in a ratio of 1:2 by weight. It was then loaded

115 into the previously prepared matrix to capture the detail of the mucogingival tissue contour. The

116 impression was allowed an intraoral setting time of 3 minutes. With the impression material with

117 opaque agent loaded matrix in place, a scan of the maxillary arch and dentition was obtained with

118 the NewTom ${ }^{\circledR}$ CBCT machine, in the Radiology Department, Peking University School and

119 Hospital of Stomatology. All the scans were taken with $0.125 \mathrm{~mm}$ slice thickness, $8 \times 8 \mathrm{~cm}$ field

120 of view size and 360 degrees with the same imaging unit (NewTom ${ }^{\circledR}$ ) and pixel size of $0.125 \mathrm{~mm}$,

121 exposure parameters: 15 seconds, $110 \mathrm{kVp}$, and $17 \mathrm{~mA}$. The DICOM (Digital Imaging and 
122 Communications in Medicine) data was generated and transferred to a volumetric imaging

123 software (Mimics 17.0, Materialise) in which three-dimensional reconstruction was conducted and

124 the image analyses were carried out.

125 Examination of the morphology of IP

126 Twenty-seven periodontally healthy subjects consisting of 135 maxillary anterior papillae were

127 enrolled in this study. The silicone matrix with opaque agent on the surface of gingival and 128 mucogingival tissue appeared radiopaque in imaging software (Fig. 1). The cross cursor was 129 located in the middle of the two adjacent teeth of the papilla to be measured. The horizontal axis 130 was positioned across the center of two adjacent root canals in the axial plane. The vertical axis 131 was positioned across the contact area or the top of contours for the open contact cases between

132 two teeth in the coronal plane. In the sagittal plane, the vertical axis was positioned parallel to the 133 line connecting center point of two adjacent root canals. The linear distance between the cortical 134 bone and the most coronal point of the inner radiopaque surface parallel to the vertical axis

135 represented $\mathrm{PH}$. The linear distance between the facial and palatal inner radiopaque surface across 136 the alveolar bone crest represented the FLT of the papilla. The IDD was measured as the distance 137 between the two adjacent roots along the horizontal axis which across the center of the adjacent 138 root canals in axial plane. All imaging measurements were obtained by a single examiner (GY). 139 In addition, 10 CBCT imaging measurements were repeated 1 week later by the examiner in order 140 to test the intra-examiner reliability.

\section{Statistical analysis}

142 Data expressed as means \pm standard deviation (SD) were analyzed with the statistical software 143 package (IBM, SPSS Statistics 20.0, Chicago, IL). The intra-examiner reliability was tested by 144 intra-class correlation coefficient (ICC). The values of the PH, FLT, and IDD measurements 
145 among the open contact point group, complete papilla group, and deficient papilla groups were

146 tested using 1-way ANVOA and Tukey tests with the significance level at $\alpha=0.05$.

147 Results

148 The distribution of the proximal contact of the included subjects are listed in Table 1. The ICC

149 values obtained range from 0.843 to 0.985 . Two morphological forms of the gingival papillae in

150 the anterior maxillary sextant were categorized: pyramid shape and col shape, with distribution of

$15197 \%$ and 3\%, respectively (Fig. 2). The mean and SD of the PH, FLT, and IDD values obtained

152 are listed in Table 1. The mean value of IDD obtained from the complete papilla group was

153 significantly smaller than that of the deficient papilla group $(\mathrm{p}<0.05)$, Table 1 . In Table 2 , the

154 mean values of the $\mathrm{PH}$ at the proximal regions obtained range from $3.87 \pm 0.63 \mathrm{~mm}$ to $4.13 \pm 0.56$

$155 \mathrm{~mm}$. There were no statistically significant differences among the different sites $(\mathrm{p}>0.05)$. The

156 mean values of the FLT were statistically significant differences among the measured sites $(\mathrm{p}<$

157 0.05). The mean value of the IDD between central incisors was significantly larger than the IDD

158 values between central and lateral incisors $(\mathrm{p}<0.05)$.

\section{Discussion}

160 This study described a gingival tissue measurement technique based on CBCT imaging, in which

161 a mixture of opaque agent and alginate impression material was used to assist visualization of the

162 gingival tissue. The CBCT images showed the bone crest which was the bottom of the IP and

163 indirectly showed the profile of papillae aided by the opaque agent. The consistency of opaque

164 agent mixed with alginate impression material maintains adequate viscosity and adaptation to the

165 gingival surface and interdental papilla areas (Cao et al., 2015). The CBCT imaging revealed two

166 types of interdental gingival contours: pyramid shape and col shape in maxillary anterior dentition,

167 which was revealed by histological examination (Cohen,1959; Ko-Kimura et al., 2003). The mean 
$168 \mathrm{PH}$ of different interproximal sites of central incisors, central and lateral incisors, lateral incisor 169 and canine were $4.13 \pm 0.56 \mathrm{~mm}, 3.87 \pm 0.63 \mathrm{~mm}, 4.07 \pm 0.58 \mathrm{~mm}$, respectively, in accordance

170 with other reports of the literature (Chu et al., 2009; Nordland \& Tarnow, 1998; Nozawa et al.,

171 2011; Tarnow, Magner, \& Fletcher, 1992). The mean PH of the different groups in the current

172 study varied between 3.99 to $4.17 \mathrm{~mm}$ and the mean value was about $4 \mathrm{~mm}$, in accordance with

173 the two previous study(Chen et al. 2001; Cho et al., 2006) which reported that the papilla was

174 present more frequently when the $\mathrm{CP}-\mathrm{BC}$ distance was approximate $4 \mathrm{~mm}$. The present data

175 revealed that the PH may not be affected by the bone crest and contact point and the $\mathrm{PH}$ seems to

176 be similar in height between complete and deficient papilla condition. The results led to accept the

177 null hypothesis that no significant differences would be found among different conditions of tooth

178 contact and presence of IP. The finding was a little different with a previous study (de Santana, de

179 Miranda, \& de Santana, 2017), which founded that the dimensions of the IP are significantly

180 increased in teeth without a contact point in comparison with their contra-lateral controls with a

181 contact point in periodontally healthy subjects. The difference may be explained by the

182 measurement method of $\mathrm{PH}$, the present study measured the distance between the bone crest and

183 papilla tip based on CBCT imaging, while de Santana et al (2017) employed the horizontal line

184 (ZL) connecting the gingival zenith from the adjacent teeth on silicon impressions as the base of

185 IP. Despite the different results, the two study may present a new understanding of the role of the

186 anatomic variables to determine the presence and size of the IP. As the dimension of interdental

187 gingiva appears to be genetically determined (Müller \& Eger, 2002). Furthermore, well designed,

188 adequately controlled investigations in the subject are highly warranted.

189 The FLT of the papilla base was thicker in the deficient papilla group than in the open CP and

190 complete papilla group (mean $9.01 \mathrm{~mm}$ versus $8.67 \mathrm{~mm}, 8.70 \mathrm{~mm}$ ), but the differences were not 
191 statistically significant among the groups (Table 1). The results indicated that the FLT does not

192 appear to have any effect on the papilla presence or PH. The findings were in accordance with a 193 previous study which reported the measurement of the FLT of the papilla as the distance between

194 the facial and lingual mid-point of a line connecting the most apical margins of the two maxillary 195 central incisors in study cast (Kim et al., 2011). The FLT assessment level at the proximal bone 196 crest goes along with the proximal cement-enamel junctions of the maxillary anterior teeth $(A s h$, 197 1993). Consequently, the FLT was highly correlated with the facial-lingual dimension of the 198 maxillary anterior teeth (Table 2).

The IDD of the papilla was significantly narrower in the complete papilla group than in the 200 deficient papilla group with the mean values of $2.07 \mathrm{~mm}$ and $1.81 \mathrm{~mm}$, respectively $(\mathrm{p}<0.05)$

201 (Table 1). Results of some previous studies (Cho et al., 2006; Martegani, 2007; Kim et al., 2011; 202 Kolte, Kolte, \& Mishra, 2014) suggested that the IDD is a significant predictor for papilla presence. However, data from other investigations reported that IDD was not a significant factor on 204 multivariate analysis along with controlling for age and CP-BC (Chang, 2008; Chow et al., 2010; Chen et al., 2010). One study even found that there was no association between IDD and papilla presence (Chow et al., 2010). In fact, the effect of an increase in IDD on papilla presence became more prominent with increased CP-BC (Kim et al., 2011). The differences in periodontal status of the subjects and methods among these studies make direct comparisons difficult (Chow et al., 2010). Thus, The relationship of IDD and presence of IP needs further investigation. The IDD of

210 the papilla between the central incisors was wider than that of the papilla between the central and

211 lateral incisor $(\mathrm{p}<0.05)$. Owing to the difference in the IDD of the papilla, the mesio-distal 212 diameter of the crown portion of the maxillary central incisor is relatively wider than that of the 213 maxillary lateral incisor (Ash, 1993). 
214 Lack of volumetric calculation of the IP space and existed was the limitation of this study.

215 Another limitation is the small sample size that needs to be addressed and will be corrected in a

216 future study to validate the findings and results.

\section{Conclusions}

218 Within the limitations of this study, the results demonstrated that the CBCT with indirect soft

219 tissue imaging technique is feasible to obtain a clear three-dimensional profile of the interproximal

220 soft and hard tissue of maxillary anterior region. The conditions of tooth contact between upper

221 anterior sextants may not influence the presence and dimension of the IP spatial structure in young

222 Chinese adults with periodontally healthy gingival tissue. This finding may provide a new insight

223 in the relationships of the interdental papilla with other anatomic factors for the diagnosis and

224 treatment planning of clinical cases involving IP region.

\section{Acknowledgements}

226 We would like to thank Dr. Dianne Gan for her help to prepare this manuscript.

227

228

229

230

231

232

\section{References}

Ash M. 1993. The Permanent Maxillary Incisors. In: Ash MM, editor. Wheeler's dental anatomy, physiology and occlusion. Philadelphia: W.B. Saunders; 1993, 128-49.

Cao J, Hu WJ, Zhang H, Liu DG, Le D, Yang G, Chung KH. 2015. A novel technique for measurement of dentogingival tissue by cone beam computed tomography. Oral Surgery, Oral Medicine, Oral Pathology and Oral Radiology 119:e82-e87 DOI: 10.1016/j.oooo.2014.10.022. 
236 Chang LC. 2008. Assessment of parameters affecting the presence of the central papilla using a

237 non-invasive radiographic method. Journal of Periodontology 79:603-609 DOI:

$238 \quad 10.1902 /$ jop.2008.070376.

239 Chen MC, Liao YF, Chan CP, Ku YC, Pan WL, Tu YK. 2010. Factors influencing the presence

240 of interproximal dental papillae between maxillary anterior teeth. Journal of Periodontology 81:

241 318-324 DOI: 10.1902/jop.2009.090361.

242 Cho HS, Jang HS, Kim DK, Park JC, Kim HJ, Choi SH, Kim CK, Kim BO. 2006. The effect

243 of interproximal distance between roots on the existence of interdental papillae according to the

244 distance from the contact point to the alveolar crest. Journal of Periodontology 77:1651-1657

245 DOI: $10.1902 /$ jop.2006.060023.

246 Chow YC, Eber RM, Tsao, YP, Shotwell JL, Wang HL. 2010. Factors associated with the

247 appearance of gingival papillae. Journal of Clinical Periodontology 37:719-727 DOI:

248 10.1111/j.1600-051X.2010.01594.x.

249 Chu SJ, Tarnow DP, Tan JHP, Stappert CF. 2009. Papilla proportions in the maxillary anterior 250 dentition. International Journal of Periodontics \& Restorative Dentistry 29:385-393.

251 Cohen B. 1959. Morphological factors in the pathogenesis of periodontal disease. British Dental 252 Journal 107:31-39.

de Santana RB, de Miranda JLC, de Santana CMM. 2017. The relationship between open

254 versus normal contact point and inter-proximal papilla dimensions in periodontally healthy young 255 adults: A controlled clinical trial. Journal of Clinical Periodontology 44: 1164-1171 DOI: $256 \quad 10.1111 /$ jcpe. 12795. 
257 Gastaldo JF, Cury PR, Sendyk WR. 2004. Effect of the vertical and horizontal distances

258 between adjacent implants and between a tooth and an implant on the incidence of interproximal

259 papilla. Journal of Periodontology 75:1242-1246.

260 Guerrero ME, Jacobs R, Loubele M, Schutyser F, Suetens P, van Steenberghe D. 2006. State-

261 of-the-art on cone beam CT imaging for preoperative planning of implant placement. Clinical oral

262 investigations 10:1-7 DOI: 10.1007/s00784-005-0031-2.

263 Januario AL, Barriviera M, Duarte WR. 2008. Soft tissue cone-beam computed tomography:

264 A novel method for the measurement of gingival tissue and the dimensions of the dentogingival

265 unit. Journal of Esthetic and Restorative Dentistry 20:366-373 DOI: 10.1111/j.1708-

$266 \quad 8240.2008 .00210 . x$.

267 Kim SA, Choi SS, Byun SJ, Chang M. 2011. Analysis of the embrasure dimensions between

268 maxillary central incisors in relation to the topography of the interdental papilla. Journal of

269 Periodontal \& Implant Science 41:273-278. DOI: 10.5051/jpis.2011.41.6.273.

270 Ko-Kimura N, Kimura-Hayashi M, Yamaguchi M, Ikeda T, Meguro D, Kanekawa M, Kasai

271 K. 2003. Some factors associated with open gingival embrasures following orthodontic

272 treatment. Australian Orthodontic Journal 19:19-24.

273 Kolte AP, Kolte RA, Mishra PR. 2014. Dimensional influence of interproximal areas on

274 existence of interdental papillae. Journal of Periodontology 85:795-801 DOI:

275 10.1902/jop.2013.130361.

276 Martegani P, Silvestri M, Mascarello F, Scipioni T, Ghezzi C, Rota C, Cattaneo V. 2007.

277 Morphometric study of the interproximal unit in the esthetic region to correlate anatomic variables 
278 affecting the aspect of soft tissue embrasure space. Journal of Periodontology 78:2260-2265 DOI:

279 10.1902/jop.2007.060517.

280 Müller HP, Eger T. 2002. Masticatory mucosa and periodontal phenotype: A review. 281 International Journal of Periodontics \& Restorative Dentistry 22:172-183.

282 Nordland WP, Tarnow DP. 1998. A classification system for loss of papillary height. Journal of 283 Periodontology 69:1124-1126.

284 Nozawa T, Yamaguchi S, Ookame Y, Shimada K, Tanaka K, Ito K. 2011. The distances 285 between the facial and palatal papillae in the maxillary anterior dentition. European Journal of 286 Esthetic Dentistry 6:88-93.

287 Ogawa M, Katagiri S, Koyanagi T, Maekawa S, Shiba T, Ohsugi Y, Takeuchi Y, Ikawa T, 288 Takeuchi S, Sekiuchi T, Arai Y, Kazama R, Wakabayashi N, Izumi Y, Iwata T. 2020. 289 Accuracy of cone beam computed tomography in evaluation of palatal mucosa thickness. Journal 290 of Clinical Periodontology 47:479-488 DOI: 10.1111/jcpe.13254.

291 Scarfe WC, Farman AG, Sukovic P. 2006. Clinical applications of cone-beam computed 292 tomography in dental practice. Journal of Canadian Dental Association 72:75-80.

293 Tarnow DP, Magner AW, Fletcher P. 1992. The effects of the distance from the contact point 294 to the crest of bone on the presence or absence of the interproximal dental papilla. Journal of 295 Periodontology 63:995-996 DOI: 10.1902/jop.1992.63.12.995.

296 Vera C, De Kok IJ, Reinhold D, Limpiphipatanakorn P, Yap AKW, Tyndall D, Cooper LF. 297 2012. Evaluation of buccal alveolar bone dimension of maxillary anterior and premolar teeth: A 
298 cone beam computed tomography investigation. International Journal of Oral \& Maxillofacial 299 Implants 27:1514-1519. 


\section{Table $\mathbf{1}$ (on next page)}

Results of interdental papilla measurements

All measurements are expressed as the mean \pm standard deviation $(\mathrm{mm})$. Data with the same superscripts represent statistically significant differences, $p<0.05$. 
1 TABLE 1 Results of interdental papilla measurements

2

\begin{tabular}{lcccc}
\hline Group & Napilla & $\begin{array}{c}\text { Facial-Lingual } \\
\text { Thickness }\end{array}$ & $\begin{array}{c}\text { Interdental } \\
\text { Distance }\end{array}$ \\
\hline Open Contact Point & 10 & $4.17 \pm 0.51$ & $8.70 \pm 0.87$ & $2.05 \pm 0.55$ \\
Complete Papilla & 110 & $3.99 \pm 0.61$ & $8.67 \pm 0.92$ & $1.81 \pm 0.39^{\dagger}$ \\
Deficient Papilla & 15 & $3.99 \pm 0.62$ & $9.01 \pm 0.81$ & $2.07 \pm 0.52^{\dagger}$ \\
\hline
\end{tabular}

3

4 All measurements are expressed as the mean \pm standard deviation $(\mathrm{mm})$.

5 Data with the same superscripts represent statistically significant differences, $p<0.05$.

6

7

8

9

10 


\section{Table 2 (on next page)}

Results of gingival tissue measurements at different interdental sites

All measurements are expressed as the mean \pm standard deviation $(\mathrm{mm})$. Data with the same superscripts represent statistically significant differences, $p<0.05$. 
2 TABLE 2 Results of gingival tissue measurements at different interdental sites

\begin{tabular}{lccc}
\hline Measurement & $\begin{array}{c}\text { Between } \\
\text { Central } \\
\text { Incisors }\end{array}$ & $\begin{array}{c}\text { Between } \\
\text { Central and Lateral } \\
\text { Incisors }\end{array}$ & $\begin{array}{c}\text { Between } \\
\text { Lateral Incisor and } \\
\text { Canine }\end{array}$ \\
\hline $\begin{array}{l}\text { Papilla Height } \\
\text { Facial-Lingual } \\
\text { Thickness }\end{array}$ & $4.13 \pm 0.56$ & $3.87 \pm 0.63$ & $4.07 \pm 0.58$ \\
Interdental Distance & $2.04 \pm 0.50^{\dagger}$ & $8.20 \pm 0.76^{\dagger}$ & $8.89 \pm 0.77^{\dagger}$ \\
\hline
\end{tabular}

3

4 All measurements are expressed as the mean \pm standard deviation $(\mathrm{mm})$.

5 Data with the same superscripts represent statistically significant differences, $p<0.05$.

6

7 
Figure 1

Representative maxillary sextant group

A) No contact point; B) Complete papilla; C) Deficient papilla
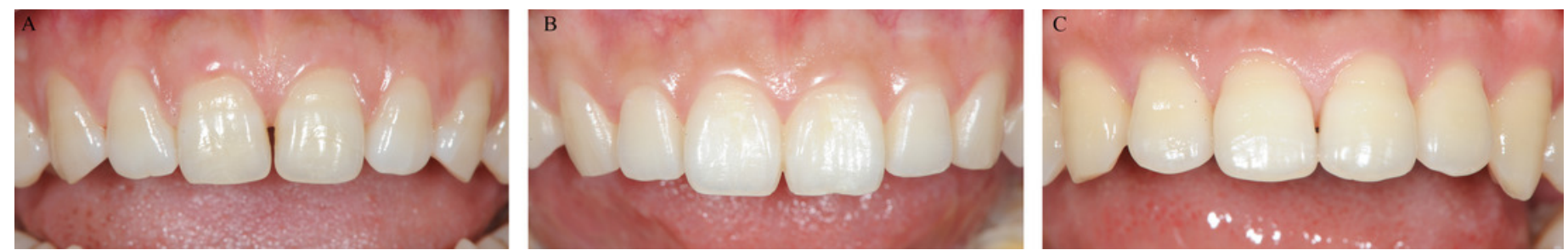


\section{Figure 2}

The sagittal axis of cone beam computed tomography imaging measurements of height and width of the interdental papilla

The sagittal and axial plane of cone beam computed tomography imaging measurements of PH, FLT and IDD. ( A) Schematic drawing of cone beam computed tomography image to show the pyramid shape of the interdental papilla morphology using radiopaque impression technique. (B) The pyramid shape of the interdental papilla image and the measurement of PH and FLT in sagittal plane. (C) The col shape of the interdental papilla image and the measurement of PH and FLT in sagittal plane. (D) The measurement of IDD in axial plane.

PH: papillae height; FLT: facial-lingual thickness; IDD: interdental distance

A

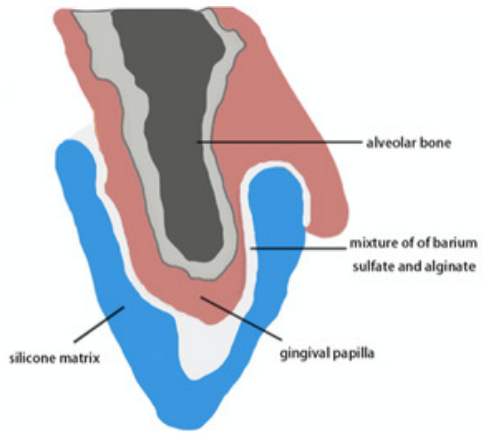

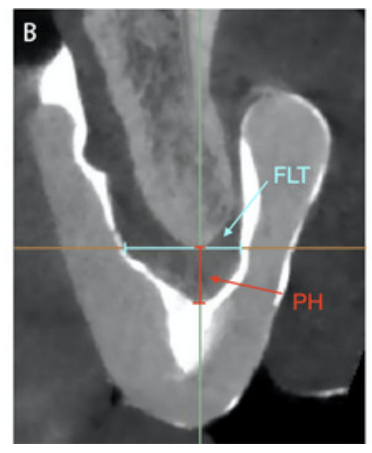

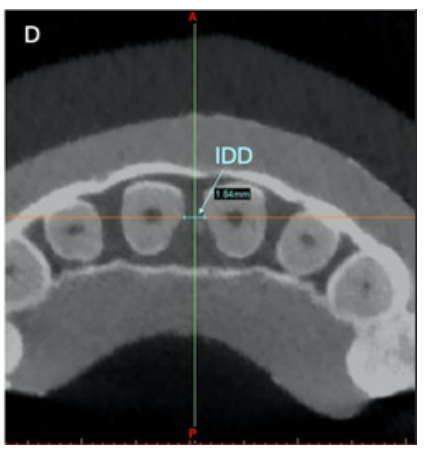

